\title{
COMMISSION 45: SPECTRAL CLASSIFICATIONS AND \\ MULTI-BAND COLOUR INDICES \\ (CLASSIFICATIONS SPECTRALES ET INDICES DE COULEUR \\ Ȧ PLUSIEURS BANDES)
}

Report of Meetings

President: B. E. Westerlund.

SECRETARY: B. Hauck.

\section{Administrative Session, 22 August 1973}

The President opened the Administrative Session and started the discussion about the usefulness to publish Commission Reports. It was concluded that these Reports are useful, in particular to the members of other Commissions, students, etc. They permit a general view of the work accomplished in the fields of astronomy and astrophysics to be obtained. In order to allow a quick consultation, but without the obligation of publishing a large list of references, the quoted publications should be followed by the number in Astronomy and Astrophysics Abstracts.

The rules concerning the organization of the Commission will be discussed by the members of the new Organizing Committee and will be communicated to all members of the Commission one year before the next General Assembly.

The following by-law proposed by the President concerning the membership in the Organizing Committee, was accepted: 'A Member of the Organizing Committee serves two consecutive terms and then retires unless he becomes President or Vice-President. For the sake of continuity the Vice-President should succeed the retiring President who then serves on the Organizing Committee one more term'.

The new President and Vice-President as well as the Organizing Committee were elected with unanimity and the fourteen new members of the Commission were approved. $K$. Nandy was elected as new member of the Group on Spectroscopic and Photometric Data.

The text on priorities and expected developments in the fields of spectral classification and multiband colour photometry was the subject of very long discussion. After some modification of the form, the text was accepted. Its essential points are:

(i) Improvement of criteria for spectral classification. It is noted that MK classification, high dispersion spectroscopy and photoelectric photometry complement each other in this refinement work; the slit spectrograms will continue to serve for monitoring photometric and objective prism surveys. Additional criteria are certainly to come from the research in the ultraviolet and infrared spectral ranges. It appears also likely that the stellar rotation will gain in importance as a specific classification parameter. Standard rotational-velocity stars will then define a system in the same way as the standard stars for spectral classification.

(ii) Calibration of the observed criteria in terms of the basic parameters mass, age and chemical composition.

Setting up a list of HR standard stars so that the various parameters of existing classification systems may be interpreted in terms of the basic astrophysical parameters.

(iii) Reclassification of HD stars into a homogeneous MK system.

(iv) Distribution of spectroscopic and photometric data, data centers.

New Organizing Committee:

President: C. O. R. Jaschek.

Vice-President: E. K. Kharadze.

Members: A. P. Cowley, B. Hauck, W. Iwanowska, P. C. Keenan, E. Mendoza V., U. Sinnerstad, V. L. Straizys, B. E. Westerlund. 


\section{Scientific Sessions, 28 August 1973}

A. THE USE OF MULTI-BAND SYSTEMS FOR CLASSIFICATION OF FAINT STARS

A. G. D. Philip: Four-Colour Photometry of Early Type Stars.

R. F. Wing: Classification of Faint Red Stars by Narrow-Band Photometry.

V. Straizys (read by V B. Nikonov): The Vilnius Photometric System for Three-Dimensional Classification of Stars and Determination of Interstellar Reddening.

\section{B. EXAMINATION OF CLASSIFICATION CRITERIA}

N. R. Walborn: The OB Stars.

C. B. Stephenson: Visual Classification Criteria for Later-Type Stars at Medium to Low Dispersion.

A. P. Cowley and N. Houk: The Michigan Spectral Catalogue, Part I and II.

$$
\text { C. DATA CENTERS }
$$

B. Hauck: Concept of a Data Center.

Joint Meeting of Commissions 37 and 45, 29 August 1973

STELLAR CONTENT OF ASSOCIATIONS AND CLUSTERS

R. Schild: The Peculiar B Stars in Orion.

R. F. Garrison: Peculiar B Stars in Scorpius, Orion and the Alpha Persei Cluster.

M. Breger: Delta Scuti Stars.

J. Dachs: On the Stellar Content of Open Clusters of the Pleiades Type.

R. Schild: The Nature of the Infrared Excesses in the Per Be Stars.

B. Hauck: Photometrical Ap Stars in Open Star Clusters.

K. C. Freeman: Some Globular Cluster Observations.

A. G. D. Philip: Bright Horizontal Branch Stars in the $\log g, \log T_{\text {eff }}$ diagram. 